Erica J. Crespi · Heather Lessig

\title{
Mothers influence offspring body size through post-oviposition maternal effects in the redbacked salamander, Plethodon cinereus
}

Received: 31 December 2002 / Accepted: 14 September 2003 / Published online: 31 October 2003

C) Springer-Verlag 2003

\begin{abstract}
In the terrestrial salamander (Plethodon cinere$u s$ ), previous work has shown that mother's body size is positively correlated to offspring size at the time of hatching even after controlling for the effects of egg size. This study was designed to determine whether maternal body size affects offspring size via pre-oviposition factors (e.g., yolk quality, jelly coat composition, or maternal genes) or post-oviposition factors (e.g., parental care behaviors, parental modification of environment). Gravid females were captured and induced to lay eggs in experimental chambers in which the environment was standardized. Fifteen clutches were exchanged, or crossfostered, between female pairs differing in body size. Ten females whose eggs were taken away and then returned served as controls for the crossing treatment. Foster mothers did not significantly differ from control mothers in the time spent with eggs, body position, or number of egg movements during brooding. Average egg mass measured midway through development was not significantly correlated to the body size of either the genetic or foster mother, but was correlated to pre-oviposition oocyte size. At hatching, offspring body length was positively correlated to egg size and the foster mother's body size. This correlation suggests that in $P$. cinereus post-oviposition maternal effects have a greater impact on offspring
\end{abstract}

E. J. Crespi $(\bowtie) \cdot$ H. Lessig

Department of Biology, University of Virginia,

238 Gilmer Hall,

P.O. Box 400327 VA 22904-4327, Charlottesville, USA

e-mail: ejcrespi@umich.edu

Tel.: +1-734-6472604

Fax: +1-734-6470884

E. J. Crespi · H. Lessig

Mountain Lake Biological Station,

Salt Pond Road,

Pembroke, VA 24136, USA

Present address:

E. J. Crespi

Department of Molecular, Cellular, and Developmental

Biology, University of Michigan,

MI 48109-1048, Ann Arbor, USA size than other maternal factors incorporated into the egg prior to oviposition. While our study showed that larger mothers moved their eggs less often and tended to spend more time in contact with their eggs, further work needs to be done to identify the specific mechanisms through which larger mothers influence the body size of their offspring. This is the first experimental demonstration of postoviposition maternal effects for any amphibian with parental care.

Keywords Maternal effects - Cross-fostering - Parental care $\cdot$ Salamanders $\cdot$ Plethodon cinereus

\section{Introduction}

A special kind of environmental effect that can influence the expression of a phenotype is the influence of maternal traits on offspring. Maternal effects are typically referred to as the non-genetic influence of mothers on their offspring (Mousseau and Fox 1998) and have been documented in all groups of vertebrates (Bernardo 1996), insects (Mousseau and Dingle 1991), and plants (Roach and Wulff 1987; Lacey 1998; Galloway 2001). Several studies have demonstrated that maternal effects can act to enhance offspring fitness (e.g., Sinervo and Doughty 1996; Donohue 1999), supporting the ecological and evolutionary importance of these effects (Kirkpatrick and Lande 1989; Bernardo 1996). Maternal environmental effects, such as egg/ovule provisioning, uterine environment, timing of reproduction, nest location, and parental care behaviors, can especially enhance offspring fitness through their effects on embryonic development (Mousseau and Fox 1998).

This study investigated the effect of maternal body size on offspring size in the redbacked salamander, Plethodon cinereus, a salamander with a completely terrestrial life cycle. Maternal effects have been studied in amphibians with aquatic reproductive modes, focusing mainly on preoviposition traits such as yolk provisioning per offspring (e.g., egg size; see review in Kaplan 1998) and oviposition 
site choice (Resetarits and Wilbur 1989; Resetarits 1996). Studying amphibian parental care, such as in the salamanders within the Plethodontidae, provides an excellent opportunity to investigate the importance of maternal effects because these species (1) allocate a large energetic investment per offspring, (2) exhibit obligate maternal care for the duration of embryonic development, (3) have long development periods that can last up to 6 months, and (4) have oviposition sites chosen by individual females (Bernardo 1996). Therefore, maternal phenotypes can affect offspring through a variety of preoviposition traits (e.g., egg size and yolk quality) and postoviposition traits (e.g., brooding behavior and oviposition site quality).

Plethodon cinereus is a particularly appropriate species to examine the ecological significance of maternal effects because it is among the best-studied terrestrial salamanders in terms of distribution, ecology, and behavior (e.g., Highton and Savage 1961; Sayler 1966; Jaeger 1972, 1979; Mathis 1990; Ng and Wilbur 1995). It is a small, slender salamander ( $\leq 50 \mathrm{~mm}$ snout-vent length) that can be found in woodlands from North Carolina to southeastern Canada and from the Atlantic coastal plain to Minnesota (Conant and Collins 1991). Females mature after 2-3 years, have small clutches of large eggs (3-14 eggs, 3.5-4.5 $\mathrm{mm}$ diameter), and display obligate maternal care (Sayler 1966).

Multivariate analyses of female reproductive traits of $P$. cinereus have shown that maternal body size has a positive affect on hatchling size after the effect of egg size is statistically removed (Crespi 2001). Beyond egg size, there are two pathways for maternal body size to affect offspring size that are not mutually exclusive. First, larger mothers may transmit factors that enhance body size of their offspring prior to oviposition, such as genes for hatchling size or yolk quality. For example, it is possible that larger mothers may provision higher quality yolk in their eggs relative to smaller females. In support of this hypothesis, larger $P$. cinereus females are associated with larger territories with more soft-bodied, high-quality prey items (Mathis 1990; Gabor 1995), which may translate into eggs with higher protein and lipid contents.

The second pathway through which larger females could positively affect their offspring size is via postoviposition effects. The eggs of amphibians are protected only by a semi-permeable jelly coat and are therefore vulnerable to continuous threats of desiccation, fungal infections, and predation throughout embryonic development (Salthe and Mecham 1974). Previous studies have shown that brooding behaviors increase egg survival via several mechanisms: reducing predation (Highton and Savage 1961; Forester 1983); increasing hydration (Forester 1984; Taigen et al. 1984); and reducing fungal or bacterial infection (Forester 1981; Austin 2000). We hypothesize that brooding behavior of larger females can also affect specific offspring traits such as offspring size. Larger females may be older and more experienced parents, thereby exhibiting maternal care behaviors that positively affect hatchling size. Alternatively, female body size may passively reduce desiccation by simply reducing the surface area of each egg that is in contact with air, thereby allowing for better-hydrated, larger offspring. For example, in the terrestrial frog Eleutherodactylus coqui, Taigen et al. (1984) found that egg mass and hatchling mass were greater when eggs were reared in contact with more moist substrates.

One method that can be used to experimentally determine the contribution of pre-oviposition and postoviposition effects on offspring is the cross-fostering technique. Cross-fostering has been used extensively in mammals and birds (Rutledge et al. 1972; Atchley and Rutledge 1980; Smith and Wettermark 1995; Roff 1998) to answer similar questions, but it has not been used with any species of salamander. Because we observed that both non-reproductive and reproductive $P$. cinereus females would brood other females' eggs in preliminary trials, we decided to use cross-fostering to determine the relative importance of pre-oviposition and post-oviposition maternal effects on offspring body size.

\section{Materials and methods}

We collected gravid female Plethodon cinereus from under rocks and $\operatorname{logs}$ in forested areas in the vicinity of Mountain Lake Biological Station, Giles County, Va., from April to May 2000. We measured snout-vent length (SVL) with digital calipers and determined clutch size and oocyte diameter by candling females with a fiber-optic light. We initially maintained females in small plastic containers with moist leaf litter at $17-19^{\circ} \mathrm{C}$ on a $15 \mathrm{~L}: 9 \mathrm{D}$ photoperiod and categorized and paired females according to body size differences and clutch size. Only clutch sizes of 7-9 eggs were used in this study to standardize for egg number and size among pairs.

To measure oocyte diameter prior to oviposition, we briefly anesthetized females by submerging them in a $0.01 \%$ phenoxyethanol solution. We positioned females on their backs on a clear plastic petri dish and then covered them with a black plastic plate with only a clear window over the female's abdomen. With a photographic flash placed underneath the dish, we took pictures that captured the transilluminated abdomen of each female. For each female, we measured the diameter of all the oocytes from these photographs and calculated the average oocyte diameter. To estimate the average preoviposition oocyte volume for each female, we assumed that the egg shape approximated a sphere and used the average oocyte radius calculated from the photographs in the equation. We could calculate pre-oviposition egg volume in only a subset of females because some photographs were not clear enough to measure egg diameter accurately.

To induce ovulation and oviposition, we used a modified protocol from Kay and Peng (1991). Females were injected with 12.5 IU of pregnant mare serum gonadotropin (Calbiochem, Inc.) in a volume of $50 \mu \mathrm{l}$ phosphate-buffered saline (PBS) into the peritoneum of the abdominal region. Females were injected $48 \mathrm{~h}$ later with $25 \mathrm{IU}$ of human chorionic gonadotropin (Chorulon, Provet, Inc.) in $50 \mu \mathrm{L}$ PBS. After the first injection, females were moved into petri dishes with moist filter paper as substrate and a half-section of 1-inch $(2.4 \mathrm{~cm})$ PVC pipe that served as a cover object. Ovulation occurred within 2-3 days after the second injection.

After we observed stereotypical brooding behavior for 10 days, entire clutches were exchanged between 30 female pairs. Ten additional females had their clutches taken away and then immediately given back to serve as controls for the effects of switching the eggs. Fourteen days after the eggs were cross-fostered, we replaced the filter paper in the petri dishes with a sphagnum pad to reduce the occurrence of bacterial and fungal infections and to 
Table 1 Mean clutch sizes with standard deviations and sample sizes for control and cross-fostered females as the developmental period progressed

\begin{tabular}{llllll}
\hline Time period & \multicolumn{2}{c}{ Control mothers } & & \multicolumn{2}{c}{ Cross-fostered mothers } \\
\cline { 2 - 3 } \cline { 5 - 6 } & Clutch size & $n$ & & Clutch size & $n$ \\
\hline Initial & $8.2 \pm 0.98$ & 10 & $7.8 \pm 0.94$ & 30 \\
Post-transfer & $7.0 \pm 1.14$ & 10 & $7.4 \pm 0.91$ & 30 \\
Mid-point (36 days) & $6.8 \pm 1.47$ & 6 & $6.5 \pm 1.64$ & 15 \\
Hatching & $5.3 \pm 1.37$ & 6 & $4.4 \pm 2.47$ & 10 \\
\hline
\end{tabular}

create a more natural brooding environment for the females. We sprayed the females with approximately $1 \mathrm{ml}$ of water periodically to maintain constant moisture. We fed the females pinhead crickets and mealworms once a week to reduce the propensity of females to eat their eggs (oophagy).

We observed females twice daily to determine whether maternal care behaviors differed between transfer and control treatments. These observations were taken between 0800 hours and 0900 hours (day) and again between $1900 \mathrm{~h}$ and $2400 \mathrm{~h}$ (night) for 5 weeks during the brooding period, resulting in a total of 32 day observations and 22 night observations. During each observation period, we recorded the following information: (1) whether or not the female was in contact with eggs, (2) whether or not eggs were moved since the last observation, and (3) the number of eggs in the clutch. The contact score was calculated as the percentage of all observations in which females were in contact with their eggs, and the egg movement score was the total number of times females were observed to rearrange or move their eggs over all observations.

Analysis of variance (ANOVA) was used to determine differences between foster mothers and control mothers in the percentage of time spent with eggs during the day and night. We used the arcsinesquare root transformation in the statistical analysis to satisfy the assumption of normality. The Mann-Whitney U-test was used to compare the number of times the eggs were moved and the number of eggs eaten between cross-foster and control mothers. These and all other statistics were calculated in SAS for Windows V. 8.1 (SAS 2000).

After 36 days of development (approximately the midpoint), we briefly removed clutches from the petri dish to measure clutch mass and average egg diameter. Clutch mass consisted of the mass of the embryos and the jelly coats surrounding them. We estimated average egg mass by dividing the total clutch mass by the number of eggs in the clutch. At the time of hatching, we measured mass and body length (head to tail) of each offspring. Total body lengths were measured because the vents were difficult to see and all tails were of full length at the time of measurement. Pearson correlation coefficients $(r)$ were calculated to reflect the relationships between average egg mass and genetic mother SVL, foster mother SVL, and pre-oviposition oocyte volume. Similarly, correlations between hatchling length and egg size (36 days), genetic mother SVL, and foster mother SVL were calculated.

Sample sizes of both the foster mothers and control mothers diminished throughout the course of the experiment due to abandonment of eggs by mothers. By 36 days of egg development (midpoint of the brooding period), 60\% of control and 50\% of foster mothers were still brooding their eggs (Table 1). The final sample

Table 2 Behavioral scores for control $(n=10)$ and cross-fostered mothers $(n=15)$ taken during both day and night observations during a 5 -week period. The mean \pm SEM are given for the Contact Score; sizes at the time of hatching were 6 control females and 10 crossfoster females. The reduction in the number of the foster females in the last measurement was not due to abandonment, but rather because we did not observe the exact hatching date of five females. Because offspring grow in length after the time of hatching, the measurements taken at this time would not be consistent with the measurements taken of the other hatchlings in the study and, therefore, were not included in the final analyses.

\section{Results}

Both transfer and control mothers ate eggs at a similar rate during the course of development (Table 1), and the number of offspring per female to survive until hatching was not significantly different between the control (mean rank 12.75) and foster mothers (mean rank 10.3) $\left(\chi^{2}=\right.$, $d f=1, P=0.4078)$. The time of observation and the crossfoster treatment did not affect the amount of time spent with eggs (day: $F_{1,19}=1.48, P=0.24$; night: $F_{1,19}=2.06$, $P=0.17$ ) or the frequency of egg movement (day: $\chi^{2}=0.88$, $d f=1, P=0.35$; night: $\chi^{2}=1.12, d f=1, P=0.29$ ) (Table 2).

After 36 days of development, egg mass was significantly correlated with estimated pre-oviposition oocyte volume ( $r=0.84, d f=1,6, P=0.009$ ), and a positive trend was observed between egg mass and foster mother's SVL $(r=0.46, d f=1,13, P=0.08)$ (Fig. 1). Egg mass did not vary with SVL of the genetic mother $(P=0.27)$. At hatching, offspring body length was significantly and positively correlated with egg mass at 36 days $(r=0.64, d f=1,8$, $P=0.05)$ and foster mothers' SVL $(r=0.77, d f=1,8$, $P<0.01$ ), but it was not correlated with genetic mothers' SVL $(r=0.07, d f=1,8, P>0.05$ ) (Fig. 1). Larger foster mothers moved their eggs fewer times than smaller mothers $(r=-0.52, d f=1,8, P=0.05)$ (Fig. 2). Larger mothers also tended to be in contact with their eggs for more time than smaller mothers $(r=0.48, d f=1,8, P=0.07)$.

\section{Discussion}

We used the cross-fostering technique successfully in this study to show that both pre- and post-oviposition maternal traits affect offspring size at hatching in Plethodon cinereus. Initial oocyte size, primarily a female body size- and clutch size-dependent trait determined prior to oviposition, was indirectly related to hatchling size via its positive relationship with egg size during development. In addition, the positive correlation between hatchling length and the foster mother's body length shows that female

\begin{tabular}{llllll}
\hline \multirow{2}{*}{ Behavior } & \multicolumn{2}{l}{ Control mothers } & & \multicolumn{2}{l}{ Cross-fostered mothers } \\
\cline { 2 - 3 } Contact score & Day & Night & Day & Night \\
\hline Egg movement score & $95 \pm 3.7$ & $93 \pm 3.9$ & $84 \pm 5.2$ & $84 \pm 3.9$ \\
\hline
\end{tabular}

the median and range (in parentheses) are given for the Egg Movement Score. Refer to text for explanation of behavioral scores 
Fig. 1 Relationships among offspring size, genetic mother's snout-vent length (SVL), and foster mother's SVL for two stages of offspring development: egg size after 36 days of development (top, $n=15$ ) and body size at hatching (bottom, $n=10$ ). Only cross-fostered clutches are represented in these graphs. Because 8 of 15 females had clear photographs of pre-oviposition oocytes, the sample size for the correlation between this variable and average egg size at 36 days was reduced. Statistically significant regression lines are shown $(P<0.05)$

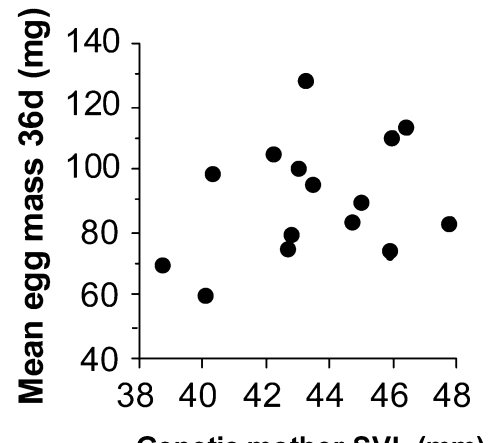

Genetic mother SVL ( $\mathrm{mm})$

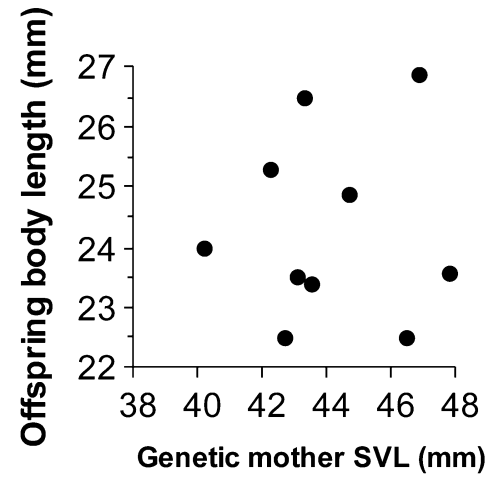

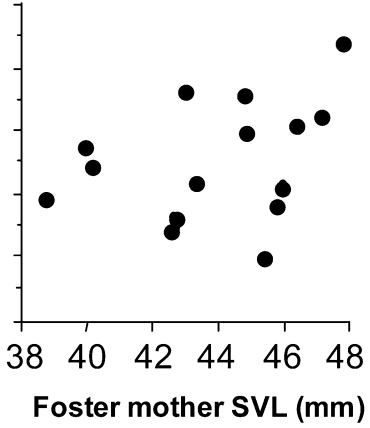
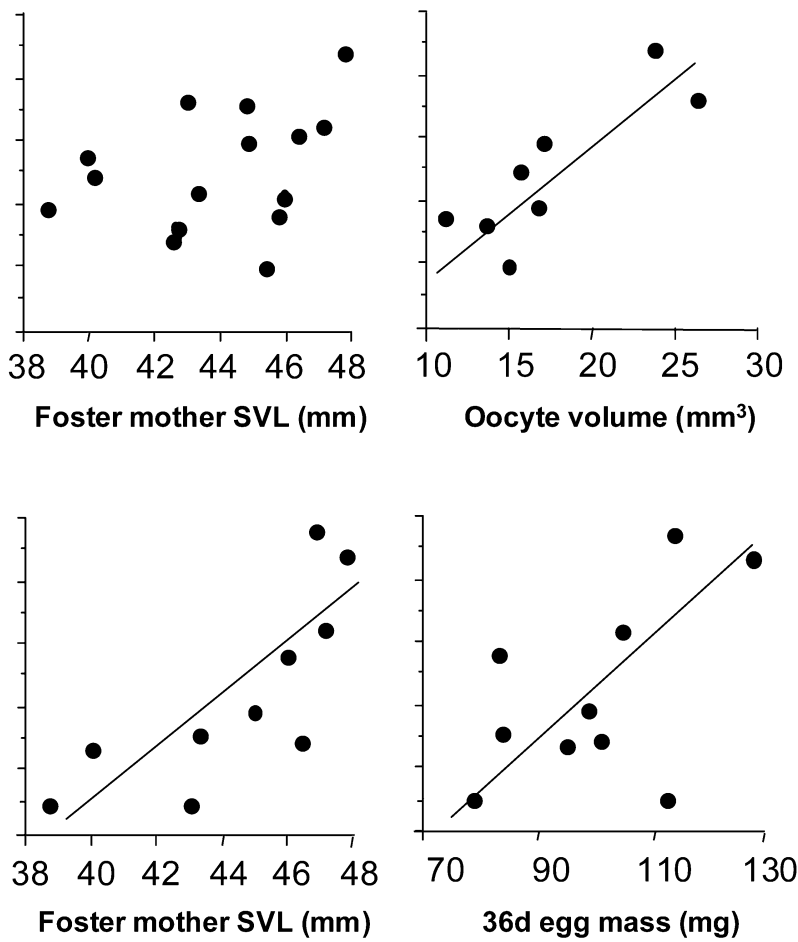
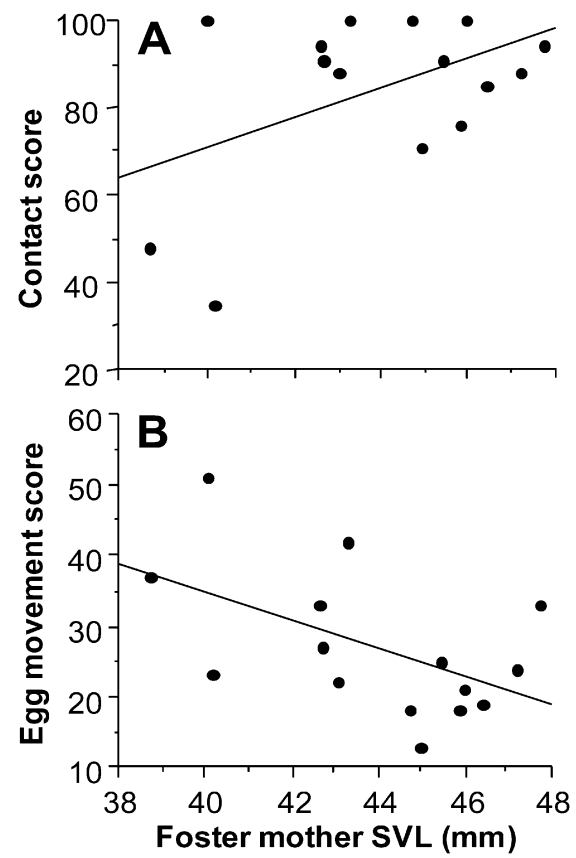

Fig. 2A, B Relationships between foster mother's snout-vent length (SVL) and brooding behaviors $(n=15)$ : A percentage of observations where females were in contact with eggs and $\mathbf{B}$ total number of times females were observed rearranging or moving eggs

body size can affect offspring size during the brooding period.

From our observations, control females who cared for their own eggs did not show significantly different brooding behaviors than females who cared for another female's eggs. This was consistent regardless of the time the behavior was observed (day or night) or the type of behavior being observed. These tests were essential to show that behavioral artifacts of the cross-fostering treatment did not confound the relationships observed among mothers, eggs, and offspring size. In addition, cross-foster and control females did not significantly differ in the rate of abandonment or in the number of eggs eaten throughout development. Abandonment and oophagy are common in field populations of P. cinereus (Crespi 2001; J. Gillette, K. Yurewicz, and H. Wilbur, personal communication) and have been documented in other lab experiments with brooding plethodontid salamanders (Highton and Savage 1961; Forester 1984). Even with reduced sample sizes, and therefore reduced statistical power, our ability to detect significant correlations in this study suggests that the effect of the parenting mother's body size is robust. Admittedly, larger sample sizes are necessary to conduct the multivariate analyses needed to fully understand the relative contribution of various maternal effects (e.g., egg size, mother's body size, and mother's behaviors) on offspring size at hatching. Overall, cross-fostering appears to be a powerful tool for future investigation of maternal effects in $P$. cinereus and other salamanders with parental care.

Although this is the first cross-fostering experiment in salamanders reported in the literature, previous crossfostering studies in birds and mammals have found that environmental effects can supersede genetic effects on offspring phenotypes (Rutledge et al. 1972; Ricklefs and Smeraski 1983), but others have found the opposite (Dhondt 1982; Smith and Wettermark 1995). It appears that the importance of the environment provided by the mother in determining various characteristics of offspring phenotype varies among the traits examined and the 
environmental conditions of a given breeding season. Indeed, previous work with $P$. cinereus suggests that the importance of female body size on offspring size depends on the environmental conditions during the brooding period (Crespi 2001). In our cross-fostering experiment, moisture, temperature, and substrate were standardized across females so that the variation in offspring size could be attributed unambiguously to aspects of the female. In a two-year field experiment, however, Crespi (2001) used multivariate analyses to show that only egg size was correlated to hatchling length in a wet year, while maternal body size completely eclipsed the egg-size effect on hatchling length in a drought year. Crespi (2001) also showed that body size at hatching was positively correlated with survival through the first year of life. While others have shown that parental care increases offspring fitness by increasing egg survival (e.g., Tilley 1972; Forester 1981), these results suggest that variation in the quality of parental care also can affect offspring fitness after hatching.

The relative importance of pre- vs post-oviposition maternal effects measured in this study is probably unique to the environmental conditions provided in the experiment. Because we used sphagnum as a substrate to maintain moisture and minimize bacterial and fungal infections, the size-dependent effect of the mothers could have been due to differential usage of the vegetation in positioning the eggs. Conducting cross-fostering experiments including other types of substrates found in oviposition sites (e.g., sandy soil or decomposing wood) or in the field could reveal varying contributions of preand post-oviposition effects in different environments. In addition, the amount of moisture and temperature can be varied to test hypotheses about the interaction of abiotic factors with size-dependent maternal effects on offspring development.

Correlations between the behavioral scores we measured and the foster mother's body size suggest that larger females may exhibit behaviors that enhance their offspring development, thereby affecting offspring body size. Larger mothers moved their eggs fewer times and tended to be in contact with their eggs more often than smaller mothers. Unfortunately, the exact mechanism through which these behaviors enhance development could not be determined by this experiment. The positive effect of contact time with eggs may be due to the reduction of desiccation or suppression of infections from microorganisms (see Forester 1984; Austin 2000). It is interesting that the number of times mothers moved eggs was negatively correlated to offspring size since vibration of eggs (simulation of gular movements of the mother) has been shown to enhance egg survival (Forester 1984). Gular vibration of eggs may enhance offspring development by increasing oxygen diffusion or minimizing the opportunity for fungi or bacteria to infect eggs (Forester 1984). Larger movements of eggs, as counted in this experiment, may represent the level of agitation of the mother in caring for specific eggs. Future studies using specific manipulations and video surveillance can be conducted to test hypotheses about the exact behaviors that affect offspring size.

In conclusion, we have shown that offspring size in $P$. cinereus is a function of both egg size and the body size of the parenting mother. Although we do not yet understand the mechanisms through which larger mothers enhance offspring size during brooding, we report the first experimental demonstration of a post-oviposition maternal effect on a specific offspring trait for any amphibian. Future investigation of $P$. cinereus using the crossfostering technique promises to reveal the complexity of maternal effects in terrestrial amphibians, as well as their ecological and evolutionary significance.

Acknowledgements We thank $\mathrm{H}$. Wilbur for his advice and guidance throughout this project. We also thank the students and faculty at Mountain Lake Biological Station during the summer of 2000 for their assistance in the execution of the experiment, especially E. Nagy, J. Watters, I. Parker-Renga, and J. McGlothlin. This study was funded by Mountain Lake Biological Station; a National Science Foundation Research Experience for Undergraduates (REU) program awarded to Mountain Lake Biological Station (DBI-973215); and a National Science Foundation Doctoral Dissertation Improvement Grant awarded to Henry Wilbur and Erica Crespi (IBN-9972674).

\section{References}

Atchley WR, Rutledge JJ (1980) Genetic components of size and shape. I. Dynamics of components of phenotypic variability and variability during ontogeny in the laboratory rat. Evolution $34: 1161-1173$

Austin RM Jr (2000) Cutaneous microbial flora and antibiosis in Plethodon ventralis: Inferences for parental care in the Plethodontidae. In: Bruce RC, Jaeger RB, Houck LD (eds) The biology of the plethodontid salamanders. Kluwer Academic/Plenum, New York

Bernardo J (1996) Maternal effects in animal zoology. Am Zool 36:83-105

Conant R, Collins JT (1991) Reptiles and amphibians of eastern/ central North America, 3rd edn. Houghton Mifflin, Boston

Crespi EJ (2001) Adaptation of reproductive strategies: Endocrine and ecological factors associated with the evolution of terrestrial breeding in amphibians. PhD Dissertation. University of Virginia, Charlottesville, Va.

Dhondt AA (1982) Heritability of blue tit tarsus length from normal and cross-fostered broods. Evolution 36:418-419

Donohue K (1999) Seed dispersal as a maternally influenced character: Mechanistic basis of maternal effects and selection on maternal characters in an annual plant. Am Nat 154:674689

Forester DC (1981) Parental care in the salamander Desmognathus ochrophaeus: Female activity pattern and trophic behavior. J Herpetol 15:29-34

Forester DC (1983) Duration of the brooding period in the mountain dusky salamander (Desmognathus ochrophaeus) and its influence on aggression toward conspecifics. Copeia 1983:10981101

Forester DC (1984) Brooding behavior by the mountain dusky salamander: Can the female's presence reduce clutch desiccation? Herpetologica 40:105-109

Gabor CR (1995) Correlational test of Mathis' hypothesis that bigger salamanders have better territories. Copeia 3:729-735

Galloway LF (2001) The effect of maternal and paternal environments on seed characters in the herbaceous plant Campanula americana (Campanulaceae). Am J Bot 88:832-840 
Highton R, Savage T (1961) Functions of the brooding behavior in the female red-backed salamander, Plethodon cinereus. Copeia 1961:95-98

Jaeger RG (1972) Food as a limited resource in competition between two species of terrestrial salamanders. J. Herpetol 8:191-194

Jaeger RG (1979) Seasonal spatial distributions of the terrestrial salamander Plethodon cinereus. Herpetologica 35:90-93

Kaplan RH (1998) Maternal effects, developmental plasticity, and life history evolution. An amphibian model. In: Mousseau TA, Fox C (eds) Maternal effects as adaptations. Oxford University Press, Oxford, pp 244-260

Kay B, Peng B (1991) Methods in cell biology, vol. 36, Xenopus laevis: Practical uses in cell and molecular biology. Academic Press, New York

Kirkpatrick M, Lande R (1989) The evolution of maternal characters. Evolution 43:485-503

Lacey EP (1998) What is an adaptive environmentally-induced parental effect? In: Mousseau TA, Fox C (eds) Maternal effects as adaptations. Oxford University Press, Oxford, pp 54-66

Mathis A (1990) Territoriality in a terrestrial salamander: The influence of resource quality and body size. Behavior 112:162175

Mousseau TA, Dingle H (1991) Maternal effects in insect life histories. Annu Rev Entomol 36:511-534

Mousseau TA, Fox CW (1998) The adaptive significance of maternal effects. Trends Ecol Evol 13:403-407

$\mathrm{Ng}$ M, Wilbur HM (1995) The cost of brooding in Plethodon cinereus. Herpetologica 51:1-8

Resetarits WJ Jr (1996) Oviposition site choice and life history evolution. Am Zool 36:205-215
Resetarits WJ Jr, Wilbur HM (1989) Choice of oviposition site in Hyla chrysoscelis: Role of predators and competitors. Ecology 70:220-228

Ricklefs RE, Smeraski CA (1983) Variation in incubation period within a population of the European starling. Auk 100:926-931

Roach DA, Wulff RD (1987) Maternal effects in plants. Annu Rev Ecol Syst 18:209-235

Roff DA (1998) The detection and measurement of maternal effects. In: Mousseau TA, Fox CW (eds) Maternal effects as adaptations. Oxford University Press, New York, pp 83-96

Rutledge JJ, Robison OW, Eisen EJ, Legates JE (1972) Dynamics of genetic and maternal effects in mice. J Anim Sci 35:911-918

Salthe SN, Mecham JS (1974) Reproductive and courtship patterns. In: Lofts B (ed) Physiology of the amphibia, vol 2. Academic Press, New York, pp 310-522

SAS Institute, Inc. (2000) SAS v.8.1 for Windows. Cary, N.C.

Sayler A (1966) The reproductive ecology of the red-backed salamander, Plethodon cinereus, in Maryland. Copeia 1966:183-193

Sinervo B, Doughty P (1996) Interactive effects of offspring size and timing of reproduction on offspring reproduction: Experimental, maternal, and quantitative genetics aspects. Evolution 50:1314-1327

Smith HG, Wettermark, KJ (1995) Heritability of nestling growth in cross-fostered European starlings Sturnus vulgaris. Genetics 141:657-665

Taigen TL, Pough FH, Stewart MM (1984) Water balance of terrestrial anuran (Eleutherodactylus coqui) eggs: Importance of parental care. Ecology 65:248-255

Tilley SG (1972) Aspects of parental care and embryonic development in Desmognathus ochrophaeus. Copeia 1972:532-540 